\title{
Identification and functional analysis of differentially expressed genes associated with cerebral ischemia/reperfusion injury through bioinformatics methods
}

\author{
XIAOLI SHAO $^{1 *}$, WANGXIAO BAO ${ }^{2 *}$, XIAOQIN HONG ${ }^{1}$, HUIHUA JIANG $^{1}$ and ZHI YU ${ }^{1}$ \\ ${ }^{1}$ Department of Neurology, Chun'an First People's Hospital, Zhejiang People's Hospital Chun'an Branch, \\ Hangzhou, Zhejiang 311700; ${ }^{2}$ Department of Neurology, First Affiliated Hospital of \\ Zhejiang University School of Medicine, Hangzhou, Zhejiang 310003, P.R. China
}

Received October 11, 2017; Accepted January 18, 2018

DOI: $10.3892 / \mathrm{mmr} .2018 .9135$

\begin{abstract}
Cerebral ischemia/reperfusion (I/R) injury results in detrimental complications. However, little is known about the underlying molecular mechanisms involved in the reperfusion stage. The aim of the present study was to identify a gene expression profile associated with cerebral ischemia/reperfusion injury. The GSE23160 dataset, which comprised data from sham control samples and post-I/R injury brain tissues that were obtained using a middle cerebral artery occlusion (MCAO) model at 2, 8 and $24 \mathrm{~h}$ post-reperfusion, was downloaded from the Gene Expression Omnibus database. The differentially expressed genes (DEGs) in the MCAO samples compared with controls were screened using the GEO2R web tool. Gene Ontology (GO) analysis and Kyoto Encyclopedia of Genes and Genomes (KEGG) pathway analysis for DEGs was performed using the online tool DAVID. Furthermore, a protein-protein interaction (PPI) network was constructed using the STRING database and Cytoscape software. In total, 32 DEGs at $2 \mathrm{~h}$ post-reperfusion, 39 DEGs at $8 \mathrm{~h}$ post-reperfusion and $91 \mathrm{DEGs}$ at $24 \mathrm{~h}$ post-reperfusion were identified, while 15 DEGs were common among all three groups. GO analysis revealed that the DEGs at all three time-points were enriched in 'chemotaxis' and 'inflammatory response' terms, while KEGG pathway analysis demonstrated that DEGs were significantly enriched in the 'chemokine signaling pathway'. Furthermore, following PPI network construction, Cxcl1 was identified as the only hub gene that was common among all three time-points. In conclusion,
\end{abstract}

Correspondence to: Dr Xiaoli Shao, Department of Neurology, Chun'an First People's Hospital, Zhejiang People's Hospital Chun'an Branch, 1869 Huanhu North Road, Hangzhou, Zhejiang 311700, P.R. China

E-mail: shaoxiaoli188@163.com

${ }^{*}$ Contributed equally

Key words: differentially expressed genes, gene ontology, cerebral ischemia/reperfusion injury, inflammatory response, chemokines the present study has demonstrated a global view of the potential molecular differences following cerebral I/R injury and may contribute to an improved understanding of the reperfusion stage, which may ultimately aid in the development of future clinical strategies.

\section{Introduction}

Globally, stroke is the second most frequent cause of mortality and the primary cause of serious long-term disability worldwide (1). Of all strokes, $87 \%$ are ischemic (2). Various mechanisms underlying ischemic stroke are driven by cell-cell interactions within brain, including excitotoxicity, calcium dysregulation, oxidative and nitrosative Stress, cortical spreading depolarizations, inflammation, necrosis, necroptosis and autophagy (3). In addition to a narrow therapeutic time window (4), ischemic stroke remains difficult to manage.

Although reperfusion has been proven to be beneficial for ischemic stroke (5), reperfusion may result in detrimental secondary damage, which is termed ischemia/reperfusion (I/R) injury. Early reperfusion of ischemic brain tissue has been associated with various negative consequences, including blood-brain barrier breakdown, which may result in cerebral edema and/or brain hemorrhage, neurovascular damage and neuronal death (6). Angiogenesis and vasculogenesis have also been detected following reperfusion (7). In addition, inflammation is induced by reperfusion injury and contributes negatively to long-term disease prognosis (8). The inflammatory response may result in subsequent oxidative injury, excitotoxicity and neuronal cell death (9). Chemokines, produced by resident microglial cells and other immune cells in the brain, contribute to the recruitment of circulating leukocytes and exaggerate the inflammatory response. Chemokines have been demonstrated to have both deleterious and beneficial roles in ischemia/reperfusion injury (10).

Microarray analysis has been previously employed to identify molecular variations in cerebral I/R injury $(11,12)$. However, gene expression profiles at different reperfusion periods have not been investigated extensively. Therefore, the present study employed a microarray dataset from the Gene Expression Omnibus (GEO) database and screened for differentially expressed genes (DEGs) between control samples and 
cerebral I/R samples at 2, 8 and $24 \mathrm{~h}$ post-reperfusion, and subsequently analyzed the functions and interactions of these DEGs. The results of the current study may aid in improving the understanding of the molecular mechanisms underlying cerebral I/R injury.

\section{Materials and methods}

Microarray data. Microarray gene expression profiles from GSE23160 (12) were obtained from the GEO database (http://www.ncbi.nlm.nih.gov/geo/), which is based on the platform of GPL6885 using Illumina MousRef-8 v2.0 Expression BeadChip (Illumina, Inc., San Diego, CA, USA). All of the samples were taken from male C57BL/6J mice (8-10 weeks). Following $2 \mathrm{~h}$ suture-induced middle cerebral artery occlusion (MCAO), the animals underwent reperfusion for 2,8 or $24 \mathrm{~h}$. Tissue extractions at 2, 8 and $24 \mathrm{~h}$ post-reperfusion and sham controls ( $\mathrm{n}=4$ per group) were included in this dataset.

Identification of DEGs. GEO2R (http://www.ncbi.nlm. nih.gov/geo/geo2r/), an R-based web application (13), was employed to analyze DEGs between MCAO samples and sham samples. $\mathrm{P}<0.05$ and $\log \mathrm{FCl} \geq 1.2$ were set as the threshold criteria to identify genes that were differentially expressed in MCAO models. Subsequently, the DEGs at 2, 8 and $24 \mathrm{~h}$ post-reperfusion were screened for subsequent analyses. A Venn diagram was produced to indicate the intersection among DEGs in the various MCAO groups using FunRich software (version 2.1.1; www.funrich.org) (14).

Functional enrichment analysis of DEGs. To identify the biological processes, cellular components, molecular functions and biological pathways that the DEGs were significantly enriched in, Gene Ontology (GO) enrichment (15) and Kyoto Encyclopedia of Genes and Genomes (KEGG) pathway analyses (16) were performed using the online tool Database for Annotation, Visualization and Integrated Discovery (DAVID; http://david.abcc.ncifcrf.gov/). $\mathrm{P}<0.05$ was considered to indicate a significantly enriched term or pathway, which was calculated using a hypergeometric test. Heat map illustration of DEGs was performed with heat map illustrator software (version 1.0.3.7; http://hemi.biocuckoo.org) (17).

Construction of the PPI network. To further investigate the underlying molecular mechanisms of cerebral I/R injury, protein-protein interaction (PPI) networks for the DEGs were constructed using the Search Tool for the Retrieval of Interacting Genes (STRING) database (http://www.string-db.org/) (18). A combined score of $>0.4$ was selected to construct the PPI networks. The obtained PPI networks at $24 \mathrm{~h}$ post-reperfusion were subsequently visualized using Cytoscape software (version 3.5.1) (19). Finally, the topological properties of the networks at 2, 8 and $24 \mathrm{~h}$ post-reperfusion were analyzed and the degree of each node was calculated; genes with a degree $>10$ were defined as hub genes.

\section{Results}

Identification of DEGs. As demonstrated in Fig. 1, 32 DEGs at $2 \mathrm{~h}$ post-reperfusion, $39 \mathrm{DEGs}$ at $8 \mathrm{~h}$ post-reperfusion

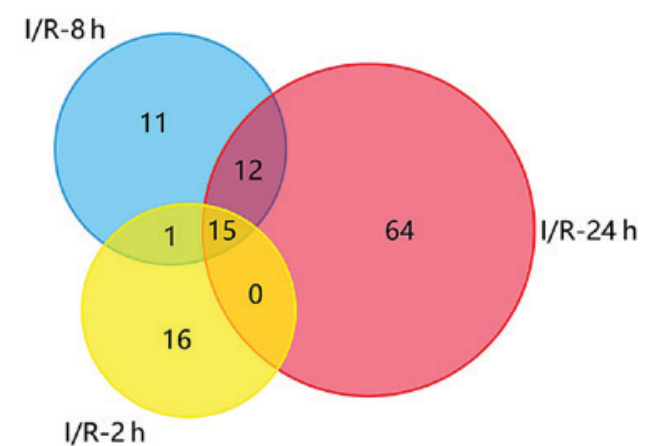

Figure 1. Venn diagram presenting the DEGs between cerebral I/R and sham control samples at 2,8 and 24 h post-reperfusion. A total of 15 DEGs were common among all three post-reperfusion time-points. DEGs, differentially expressed genes; I/R, ischemia/reperfusion.

and $91 \mathrm{DEGs}$ at $24 \mathrm{~h}$ post-reperfusion were identified in the MCAO samples compared with the controls. Among them, 15 DEGs were common to all three injury samples, including $\mathrm{C}-\mathrm{C}$ motif chemokine ligand (CCL)7, suppressor of cytokine signaling 3, CCL 4 , activating transcription factor 3 , lipocalin 2, hemoglobin $\alpha$ adult chain 1, gap junction protein $\beta 2, \mathrm{CD} 14$ antigen, CCL3, heat shock protein $1 \mathrm{~A}$, S100 calcium-binding protein A8 (calgranulin A), C-X-C motif chemokine ligand 1 (CXCL1), epithelial membrane protein 1, tissue inhibitor of metalloproteinase 1 and zinc finger protein 36 , all of which were upregulated in the MCAO samples (Table I). Heat map and PPI network analysis at 2 and $8 \mathrm{~h}$ post-reperfusion are not presented due to the small number of identified DEGs.

Bioinformatics analyses of DEGs. To further the understanding of the screened DEGs and determine their potential roles following I/R injury, GO functional and KEGG pathway enrichment analyses were performed.

A total of $51 \mathrm{GO}$ enriched terms for biological processes at 2, 8 and $24 \mathrm{~h}$ post-reperfusion were obtained. The 10 most enriched GO terms according to the P-value for 2, 8 and 24 h post-reperfusion groups are presented in Table II. Furthermore, 'chemotaxis' (GO:0006935), 'inflammatory response' (GO:0006954), 'immune response' (GO:0006955), 'G-protein coupled receptor signaling pathway' (GO:0007186), 'response to toxic substance' (GO:0009636), 'neutrophil chemotaxis' (GO:0030593), 'positive regulation of tumor necrosis factor production' (GO:0032760), 'positive regulation of GTPase activity' (GO:0043547), 'lymphocyte chemotaxis' (GO:0048247), 'positive regulation of inflammatory response' (GO:0050729), 'cell chemotaxis' (GO:0060326), 'chemokine-mediated signaling pathway' (GO:0070098), 'positive regulation of ERK1 and ERK2 cascade' (GO:0070374), 'cellular response to interferon-gamma' (GO:0071346), 'cellular response to interleukin-1' (GO:0071347), 'monocyte chemotaxis' (GO:0002548) and 'cellular response to tumor necrosis factor' (GO:0071356) were significantly enriched at all three post-reperfusion time-points (2, 8 and $24 \mathrm{~h})$.

Additionally, DEGs were enriched in various GO cellular component terms; at 2 and $8 \mathrm{~h}$ post-reperfusion, DEGs were enriched in 'extracellular region' (GO:0005576), while 
Table I. DEGs between cerebral I/R and sham control samples that were common among 2, 8 and 24 h post-reperfusion time-points.

\begin{tabular}{|c|c|c|c|c|c|c|c|}
\hline \multirow{3}{*}{$\begin{array}{l}\text { Gene } \\
\text { symbol }\end{array}$} & \multirow[b]{3}{*}{ Gene name } & \multicolumn{6}{|c|}{ Post-reperfusion time-point } \\
\hline & & \multicolumn{2}{|c|}{$2 \mathrm{~h}$} & \multicolumn{2}{|c|}{$8 \mathrm{~h}$} & \multicolumn{2}{|c|}{$24 \mathrm{~h}$} \\
\hline & & P-value & Log FC & $\mathrm{P}$-value & Log FC & $\mathrm{P}$-value & Log FC \\
\hline ATF3 & Activating transcription factor 3 & $5.01 \times 10^{-6}$ & 2.026964 & $3.56 \times 10^{-5}$ & 1.692171 & $7.49 \times 10^{-3}$ & 1.591308 \\
\hline CCL3 & $\mathrm{C}-\mathrm{C}$ motif chemokine ligand 3 & $5.13 \times 10^{-10}$ & 3.259239 & $7.05 \times 10^{-6}$ & 3.291652 & $4.81 \times 10^{-4}$ & 2.300334 \\
\hline CCL4 & $\mathrm{C}-\mathrm{C}$ motif chemokine ligand 4 & $1.32 \times 10^{-10}$ & 4.429954 & $1.29 \times 10^{-6}$ & 4.47245 & $5.83 \times 10^{-5}$ & 3.679271 \\
\hline CCL7 & C-C motif chemokine ligand 7 & $2.18 \times 10^{-5}$ & 1.220192 & $7.63 \times 10^{-6}$ & 1.426973 & $4.00 \times 10^{-3}$ & 1.707457 \\
\hline CD14 & CD14 antigen & $1.38 \times 10^{-7}$ & 1.757282 & $5.92 \times 10^{-7}$ & 2.578573 & $2.56 \times 10^{-3}$ & 2.353608 \\
\hline CXCL1 & $\mathrm{C}-\mathrm{X}-\mathrm{C}$ motif chemokine ligand 1 & $3.29 \times 10^{-6}$ & 1.806643 & $4.81 \times 10^{-8}$ & 2.878735 & $1.43 \times 10^{-3}$ & 2.596958 \\
\hline EMP1 & Epithelial membrane protein 1 & $1.68 \times 10^{-6}$ & 1.688657 & $6.78 \times 10^{-7}$ & 1.383669 & $1.89 \times 10^{-3}$ & 2.161470 \\
\hline GJB2 & Gap junction protein $\beta 2$ & $3.16 \times 10^{-8}$ & 1.999569 & $1.15 \times 10^{-7}$ & 1.717101 & $9.13 \times 10^{-5}$ & 1.822626 \\
\hline HBA-A1 & Hemoglobin $\alpha$, adult chain 1 & $5.38 \times 10^{-7}$ & 2.436023 & $2.15 \times 10^{-8}$ & 3.757753 & $4.48 \times 10^{-4}$ & 3.807134 \\
\hline HSPA1A & Heat shock protein $1 \mathrm{~A}$ & $1.69 \times 10^{-6}$ & 3.054788 & $3.80 \times 10^{-5}$ & 2.545466 & $1.59 \times 10^{-2}$ & 1.299110 \\
\hline LCN2 & Lipocalin 2 & $4.06 \times 10^{-9}$ & 1.312245 & $4.47 \times 10^{-15}$ & 3.250233 & $1.72 \times 10^{-5}$ & 3.408973 \\
\hline S100A8 & $\begin{array}{l}\text { S100 calcium-binding protein A8 } \\
\text { (calgranulin A) }\end{array}$ & $2.29 \times 10^{-6}$ & 1.240885 & $1.66 \times 10^{-6}$ & 1.655962 & $1.90 \times 10^{-3}$ & 3.780381 \\
\hline SOCS3 & Suppressor of cytokine signaling 3 & $3.54 \times 10^{-8}$ & 2.009771 & $1.74 \times 10^{-8}$ & 2.004998 & $5.48 \times 10^{-4}$ & 2.361302 \\
\hline TIMP1 & Tissue inhibitor of metalloproteinase 1 & $1.02 \times 10^{-8}$ & 2.376190 & $3.61 \times 10^{-18}$ & 3.403190 & $1.52 \times 10^{-5}$ & 3.874533 \\
\hline ZFP36 & Zinc finger protein 36 & $2.01 \times 10^{-5}$ & 1.595428 & $6.26 \times 10^{-7}$ & 1.404682 & $1.17 \times 10^{-2}$ & 1.330419 \\
\hline
\end{tabular}

Table II. Top 10 enriched GO biological process terms for DEGs between cerebral ischemia/reperfusion and sham control samples.

A, Top 10 enriched GO biological process terms for DEGs at $2 \mathrm{~h}$ post-reperfusion

\begin{tabular}{|c|c|c|c|c|c|}
\hline GO ID & GO term & Count & $\%$ & P-value & Genes \\
\hline GO:0030593 & Neutrophil chemotaxis & 5 & 15.63 & $5.65 \times 10^{-6}$ & CXCL1, CCL3, S100A8, CCL4, CCL7 \\
\hline GO:0032570 & Response to progesterone & 4 & 12.50 & $1.80 \times 10^{-5}$ & FOS, OXT, FOSB, GJB2 \\
\hline GO:0006954 & Inflammatory response & 7 & 21.88 & $2.24 \times 10^{-5}$ & $\begin{array}{l}\text { CXCL1, CCL3, S100A8, CCL4, CCL7, } \\
\text { CD14, IL1A }\end{array}$ \\
\hline GO:0045944 & $\begin{array}{l}\text { Positive regulation of transcription } \\
\text { from RNA polymerase II promoter }\end{array}$ & 10 & 31.25 & $2.99 \times 10^{-5}$ & $\begin{array}{l}\text { FOS, CCL3, EGR2, ATF3, EGR4, FOSB, } \\
\text { NPAS4, JUNB, IL1A, CYR61 }\end{array}$ \\
\hline GO:0071356 & $\begin{array}{l}\text { Cellular response to tumor } \\
\text { necrosis factor }\end{array}$ & 5 & 15.63 & $3.59 \times 10^{-5}$ & LCN2, ZFP36, CCL3, CCL4, CCL7 \\
\hline GO:2000503 & $\begin{array}{l}\text { Positive regulation of natural killer } \\
\text { cell chemotaxis }\end{array}$ & 3 & 9.38 & $4.25 \times 10^{-5}$ & CCL3, CCL4, CCL7 \\
\hline GO:0006935 & Chemotaxis & 5 & 15.63 & $4.73 \times 10^{-5}$ & CCL3, S100A8, CCL4, CCL7, CYR61 \\
\hline GO:0051591 & Response to cAMP & 4 & 12.50 & $8.99 \times 10^{-5}$ & FOS, OXT, FOSB, JUNB \\
\hline GO:0070098 & $\begin{array}{l}\text { Chemokine-mediated signaling } \\
\text { pathway }\end{array}$ & 4 & 12.50 & $1.13 \times 10^{-4}$ & CXCL1, CCL3, CCL4, CCL7 \\
\hline GO:0050729 & $\begin{array}{l}\text { Positive regulation of } \\
\text { inflammatory response }\end{array}$ & 4 & 12.50 & $1.69 \times 10^{-4}$ & CCL3, S100A8, CCL4, CCL7 \\
\hline
\end{tabular}

B, Top 10 enriched GO biological process terms for DEGs at $8 \mathrm{~h}$ post-reperfusion

\begin{tabular}{lccccc}
\hline GO ID & GO term & Count & $\%$ & P-value & Genes \\
\hline GO:0030593 Neutrophil chemotaxis & 10 & 25.64 & $8.69 \times 10^{-15}$ & $\begin{array}{l}\text { CXCL1, CCL12, CCL3, S100A8, LGALS3, } \\
\text { CCL9, CCL4, CCL7, FCGR3, CCL17 }\end{array}$
\end{tabular}


Table II. Continued.

B, Top 10 enriched GO biological process terms for DEGs at $8 \mathrm{~h}$ post-reperfusion

\begin{tabular}{|c|c|c|c|c|c|}
\hline GO ID & GO term & Count & $\%$ & P-value & Genes \\
\hline GO:0002548 & Monocyte chemotaxis & 8 & 20.51 & $1.19 \times 10^{-12}$ & $\begin{array}{l}\text { CCL12, CCL3, FLT1, LGALS3, CCL9, } \\
\text { CCL4, CCL7, CCL17 }\end{array}$ \\
\hline GO:0070098 & $\begin{array}{l}\text { Chemokine-mediated signaling } \\
\text { pathway }\end{array}$ & 7 & 17.95 & $1.09 \times 10^{-9}$ & $\begin{array}{l}\text { CXCL1, CCL12, CCL3, CCL9, CCL4, } \\
\text { CCL7, CCL17 }\end{array}$ \\
\hline GO:0071356 & $\begin{array}{l}\text { Cellular response to tumor } \\
\text { necrosis factor }\end{array}$ & 8 & 20.51 & $1.83 \times 10^{-9}$ & $\begin{array}{l}\text { LCN2, ZFP36, CCL12, CCL3, CCL9, CCL4, } \\
\text { CCL7, CCL17 }\end{array}$ \\
\hline GO:0050729 & $\begin{array}{l}\text { Positive regulation of } \\
\text { inflammatory response }\end{array}$ & 7 & 17.95 & $2.52 \times 10^{-9}$ & $\begin{array}{l}\text { CCL12, CCL3, S100A8, CCL9, TLR2, } \\
\text { CCL4, CCL7 }\end{array}$ \\
\hline GO:0006935 & Chemotaxis & 8 & 20.51 & $3.01 \times 10^{-9}$ & $\begin{array}{l}\text { CCL12, CCL3, FLT1, S100A8, CCL9, CCL4, } \\
\text { CCL7, CCL17 }\end{array}$ \\
\hline GO:0071346 & $\begin{array}{l}\text { Cellular response to } \\
\text { interferon-gamma }\end{array}$ & 7 & 17.95 & $4.02 \times 10^{-9}$ & $\begin{array}{l}\text { CCL12, CCL3, CCL9, CCL4, GBP2, CCL7, } \\
\text { CCL17 }\end{array}$ \\
\hline GO:0048247 & Lymphocyte chemotaxis & 6 & 15.38 & $5.34 \times 10^{-9}$ & CCL12, CCL3, CCL9, CCL4, CCL7, CCL17 \\
\hline GO:0071347 & Cellular response to interleukin-1 & 7 & 17.95 & $1.09 \times 10^{-8}$ & $\begin{array}{l}\text { LCN2, CCL12, CCL3, CCL9, CCL4, CCL7, } \\
\text { CCL17 }\end{array}$ \\
\hline GO:0006954 & Inflammatory response & 10 & 25.64 & $1.76 \times 10^{-8}$ & $\begin{array}{l}\text { CXCL1, CCL12, CCL3, S100A8, CCL9, } \\
\text { TLR2, CCL4, CCL7, CD14, CCL17 }\end{array}$ \\
\hline
\end{tabular}

C, Top 10 enriched GO biological process terms for DEGs at $24 \mathrm{~h}$ post-reperfusion

\begin{tabular}{|c|c|c|c|c|c|c|}
\hline GO ID & GO term & & Coun & $\%$ & P-value & Genes \\
\hline GO:0006954 & Inflammatory response & & 21 & 22.83 & $1.99 \times 10^{-16}$ & $\begin{array}{l}\text { CXCL1, CCL3, CCL2, S100A8, CCL21C, } \\
\text { S100A9, CCL9, TLR2, CCL21A, PF4, FPR2, } \\
\text { IDO1, CCL4, CCL7, CXCL10, SLC11A1, } \\
\text { CYBA, CCL12, CHIL3, CD14, SPP1 }\end{array}$ \\
\hline GO:0006955 & Neutrophil chemotaxis & 13 & 14.13 & $5.60 \times 10^{-16}$ & $\begin{array}{l}\text { CXCL1, } \\
\text { S100A9, }\end{array}$ & $\begin{array}{l}\text { CCL3, CCL2, S100A8, LGALS3, CCL21C, } \\
\text { CCL21A, CCL9, CCL4, CCL7, CCL12, SPP1 }\end{array}$ \\
\hline GO:0006956 & $\begin{array}{l}\text { Chemokine-mediated } \\
\text { signaling pathway }\end{array}$ & 11 & 11.96 & $1.08 \times 10^{-13}$ & $\begin{array}{l}\text { CXCL1, } \\
\text { CCL21A }\end{array}$ & $\begin{array}{l}\text { CCL12, CCL3, CCL2, CCL21C, CCL9, } \\
\text { PF4, CCL4, CCL7, CXCL10 }\end{array}$ \\
\hline GO:0006957 & Monocyte chemotaxis & 9 & 9.78 & $1.54 \times 10^{-11}$ & $\begin{array}{l}\text { CCL12, } \\
\text { CCL21A }\end{array}$ & $\begin{array}{l}\text { CL3, CCL2, LGALS3, CCL21C, CCL9, } \\
\text { CCL4, CCL7 }\end{array}$ \\
\hline GO:0006958 & $\begin{array}{l}\text { Positive regulation of } \\
\text { inflammatory response }\end{array}$ & 10 & 10.87 & $1.92 \times 10^{-11}$ & $\begin{array}{l}\text { CCL12, C } \\
\text { TLR2, CC }\end{array}$ & $\begin{array}{l}\text { CL3, CCL2, S100A8, S100A9, CCL9, TGM2, } \\
\text { CL4, CCL7 }\end{array}$ \\
\hline GO:0006959 & $\begin{array}{l}\text { Cellular response to tumor } \\
\text { necrosis factor }\end{array}$ & 11 & 11.96 & $1.39 \times 10^{-10}$ & $\begin{array}{l}\mathrm{LCN} 2, \mathrm{ZF} \\
\mathrm{CCL} 9, \mathrm{CC}\end{array}$ & $\begin{array}{l}\text { P36, CCL12, CYBA, CCL3, CCL2, CCL21C, } \\
\text { CL21A, CCL4, CCL7 }\end{array}$ \\
\hline GO:0006960 & $\begin{array}{l}\text { Cellular response to } \\
\text { interleukin-1 }\end{array}$ & 10 & 10.87 & $1.76 \times 10^{-10}$ & $\begin{array}{l}\mathrm{LCN} 2, \mathrm{CC} \\
\text { CCL21A }\end{array}$ & $\begin{array}{l}\text { CL12, CCL3, CCL2, CCL21C, CCL9, SAA3, } \\
\text { CCL4, CCL7 }\end{array}$ \\
\hline GO:0006961 & Lymphocyte chemotaxis & 8 & 8.70 & $1.96 \times 10^{-10}$ & $\begin{array}{l}\text { CCL12, } \\
\text { CCL4, CC }\end{array}$ & $\begin{array}{l}\text { CL3, CCL2, CCL21C, CCL9, CCL21A, } \\
\text { CL7 }\end{array}$ \\
\hline GO:0006962 & Chemotaxis & 11 & 11.96 & $2.80 \times 10^{-10}$ & $\begin{array}{l}\text { CCL12, } \\
\text { FPR2, CC }\end{array}$ & $\begin{array}{l}\text { CL3, CCL2, S100A8, S100A9, CCL9, PF4, } \\
L 4, \text { CCL7, CXCL10 }\end{array}$ \\
\hline GO:0006963 & $\begin{array}{l}\text { Cellular response to } \\
\text { interferon-gamma }\end{array}$ & 9 & 9.78 & $1.33 \times 10^{-9}$ & $\begin{array}{l}\text { CCL12, } \\
\text { CCL4, Gl }\end{array}$ & $\begin{array}{l}\text { CL3, CCL2, CCL21C, CCL9, CCL21A, } \\
\text { 3P2, CCL7 }\end{array}$ \\
\hline
\end{tabular}

GO, Gene Ontology; DEGs, differentially expressed genes.

'membrane' (GO:0016020) was significantly enriched at both 8 and 24 h post-reperfusion (Table III). Furthermore, Table IV indicates that DEGs were significantly enriched in 'cytokine activity' (GO:0005125) and 'chemokine activity'
(GO:0008009) GO molecular function terms at 2, 8 and $24 \mathrm{~h}$ post-reperfusion.

Table V presents the KEGG pathways that were significantly enriched in DEGs. KEGG analysis indicated that "chemokine 
Table III. GO cellular component terms for DEGs between cerebral ischemia/reperfusion and sham control samples.

A, Enriched GO cellular component terms for DEGs at $2 \mathrm{~h}$ post-reperfusion

\begin{tabular}{llcccl}
\hline GO ID & GO term & Count & $\%$ & P-value & \multicolumn{1}{c}{ Genes } \\
\hline GO:0005576 & Extracellular region & 14 & 43.75 & $9.56 \times 10^{-7}$ & CXCL1, CCL3, AVP, S100A8, PMCH, OXT, CCL4, CCL7, \\
& & & & & TIMP1, LCN2, NPTX2, IL1A, CD14, CYR61 \\
GO:0005615 & Extracellular space & 12 & 37.50 & $1.02 \times 10^{-5}$ & $\begin{array}{l}\text { LCN2, CXCL1, AVP, CCL3, S100A8, PMCH, OXT, CCL4, } \\
\text { CCL7, CD14, IL1A, TIMP1 }\end{array}$ \\
& & & & &
\end{tabular}

B, Enriched GO cellular component terms for DEGs at $8 \mathrm{~h}$ post-reperfusion

\begin{tabular}{|c|c|c|c|c|c|}
\hline GO ID & GO term & Count & $\%$ & P-value & Genes \\
\hline GO:0005615 & Extracellular space & 16 & 41.03 & $4.81 \times 10^{-8}$ & $\begin{array}{l}\text { CXCL1, CCL3, FLT1, S100A8, LGALS3, PMCH, CCL9, } \\
\text { CCL4, CCL7, TIMP1, CCL17, LCN2, CCL12, SERPINA3N, } \\
\text { DMKN, CD14 }\end{array}$ \\
\hline GO:0005576 & Extracellular region & 14 & 35.90 & $1.39 \times 10^{-5}$ & $\begin{array}{l}\text { CXCL1, CCL3, S100A8, LGALS3, PMCH, CCL9, CCL4, } \\
\text { CCL7, TIMP1, LCN2, CCL12, SERPINA3N, DMKN, CD14 }\end{array}$ \\
\hline GO:0009897 & $\begin{array}{l}\text { External side of } \\
\text { plasma membrane }\end{array}$ & 5 & 12.82 & $3.17 \times 10^{-3}$ & LGALS3, OSMR, TLR2, CD14, FCGR3 \\
\hline GO:0016020 & Membrane & 20 & 51.28 & $4.79 \times 10^{-2}$ & $\begin{array}{l}\text { GPR84, FLT1, S100A8, LGALS3, OSMR, FKBP5, MS4A6D, } \\
\text { TLR2, SLC10A6, GJB2, FCGR3, HBA-A1, CH25H, PLIN4, } \\
\text { HMOX1, ITGAD, SLC15A3, GBP2, EMP1, CD14 }\end{array}$ \\
\hline
\end{tabular}

C, Enriched GO cellular component terms for DEGs at 24 h post-reperfusion

\begin{tabular}{|c|c|c|c|c|c|}
\hline GO ID & GO term & Count & $\%$ & P-value & Genes \\
\hline GO:0009897 & $\begin{array}{l}\text { External side of } \\
\text { plasma membrane }\end{array}$ & 8 & 8.70 & $6.31 \times 10^{-4}$ & $\begin{array}{l}\text { FCGR2B, LGALS3, PDPN, CCL21C, FCGR4, TLR2, CD14, } \\
\text { CXCL10 }\end{array}$ \\
\hline GO:0048237 & $\begin{array}{l}\text { Rough endoplasmic } \\
\text { reticulum lumen }\end{array}$ & 3 & 3.26 & $4.29 \times 10^{-4}$ & LYZ2, LYZ1, CHIL3 \\
\hline GO:0009986 & Cell surface & 10 & 10.87 & $2.33 \times 10^{-3}$ & $\begin{array}{l}\text { SLC11A1, THBD, FCGR2B, LGALS3, TNFRSF12A, IFITM3, } \\
\text { FCGR4, TLR2, CD14, ANXA2 }\end{array}$ \\
\hline GO:0005886 & Plasma membrane & 34 & 36.96 & $8.00 \times 10^{-3}$ & $\begin{array}{l}\text { GPR182, GPR84, S100A8, IFITM2, TNFRSF12A, IFITM3, } \\
\text { VIM, S100A9, TLR2, CD52, FPR2, SLC11A1, P2RY6, DAB2, } \\
\text { PLIN2, HMOX1, TGM2, STRA6, CLEC4D, ANGPT2, ACTB, } \\
\text { PDPN, LILRB4A, GJB2, ANXA2, CYBA, THBD, FCGR2B, } \\
\text { HSPB1, SCN4B, RGS9, EMP3, CD14, EMP1 }\end{array}$ \\
\hline GO:0016020 & Membrane & 44 & 47.83 & $1.14 \times 10^{-2}$ & $\begin{array}{l}\text { GPR182, GPR84, GFAP, TSPO, S100A8, IFITM2, TNFRSF12A, } \\
\text { IFITM3, S100A9, TLR2, CD52, FPR2, FXYD5, GLIPR2, } \\
\text { SLC11A1, P2RY6, DAB2, PLIN2, HMOX1, CH25H, TGM1, } \\
\text { TGM2, STRA6, CLEC4D, ACTB, LGALS3, PDPN, MS4A6D, } \\
\text { LILRB4A, GJB2, ANXA2, HBA-A1, CYBA, RAB32, THBD, } \\
\text { FCGR2B, SCN4B, RGS9, EMP3, SLC15A3, GBP2, CD14, } \\
\text { EMP1, MVP }\end{array}$ \\
\hline
\end{tabular}

GO, Gene Ontology; DEGs, differentially expressed genes.

signaling pathway', 'cytokine-cytokine receptor interaction' and 'toll-like receptor signaling pathway' were significantly enriched in DEGs at 2, 8 and $24 \mathrm{~h}$ post-reperfusion. Furthermore, as demonstrated in Fig. 2, a total of 11 chemokine signaling pathway-associated genes were overexpressed in $24 \mathrm{~h}$ post-reperfusion injury samples compared with the sham control samples.
PPI network analysis. Genes with an interaction degree $>10$ in the PPI network analysis of DEGs at 2, 8 and $24 \mathrm{~h}$ post-reperfusion were defined as hub genes, which are listed in Table VI. CXCL1 was the only gene that was considered to be a hub gene at 2, 8 and 24 h post-reperfusion (Table VI). The constructed PPI network of $24 \mathrm{~h}$ post-reperfusion samples is presented in 
Table IV. Enriched GO molecular function terms for DEGs between cerebral ischemia/reperfusion and sham control samples.

A, Enriched GO molecular function terms for DEGs at $2 \mathrm{~h}$ post-reperfusion

\begin{tabular}{|c|c|c|c|c|c|}
\hline GO ID & GO term & Count & $\%$ & P-value & Genes \\
\hline GO:0005125 & Cytokine activity & 6 & 18.75 & $2.48 \times 10^{-5}$ & CXCL1, FOS, CCL3, CCL4, CD14, IL1A \\
\hline GO:0008009 & Chemokine activity & 4 & 12.50 & $6.79 \times 10^{-5}$ & FOS, CCL3, CCL4, CD14 \\
\hline GO:0000978 & $\begin{array}{l}\text { RNA polymerase II core promoter } \\
\text { proximal region sequence-specific } \\
\text { DNA binding }\end{array}$ & 6 & 18.75 & $2.84 \times 10^{-4}$ & FOS, EGR2, ATF3, FOSB, NPAS4, JUNB \\
\hline GO:0001077 & $\begin{array}{l}\text { Transcriptional activator activity, } \\
\text { RNA polymerase II core promoter } \\
\text { proximal region sequence-specific } \\
\text { binding }\end{array}$ & 5 & 15.63 & $9.82 \times 10^{-4}$ & FOS, EGR2, FOSB, NPAS4, JUNB \\
\hline GO:0003690 & Double-stranded DNA binding & 3 & 9.38 & $2.13 \times 10^{-2}$ & FOS, FOSB, JUNB \\
\hline GO:0003677 & DNA binding & 8 & 25.00 & $2.85 \times 10^{-2}$ & $\begin{array}{l}\text { ZFP36, FOS, EGR2, ATF3, EGR4, FOSB, } \\
\text { NPAS4, JUNB }\end{array}$ \\
\hline
\end{tabular}

B, Enriched GO molecular function terms for DEGs at $8 \mathrm{~h}$ post-reperfusion

\begin{tabular}{|c|c|c|c|c|c|}
\hline GO ID & GO term & Count & $\%$ & P-value & Genes \\
\hline GO:0008009 & Chemokine activity & 7 & 17.95 & $3.98 \times 10^{-10}$ & $\begin{array}{l}\text { CXCL1, CCL12, CCL3, CCL9, CCL4, CCL7, } \\
\text { CCL17 }\end{array}$ \\
\hline GO:0005125 & Cytokine activity & 8 & 20.51 & $1.54 \times 10^{-7}$ & $\begin{array}{l}\text { CXCL1, CCL12, CCL3, CCL9, CCL4, CCL7, } \\
\text { CCL17, TIMP1 }\end{array}$ \\
\hline GO:0048020 & CCR chemokine receptor binding & 4 & 10.26 & $2.14 \times 10^{-5}$ & CCL3, CCL9, CCL4, CCL17 \\
\hline
\end{tabular}

C, Enriched GO molecular function terms for DEGs at $24 \mathrm{~h}$ post-reperfusion

\begin{tabular}{|c|c|c|c|c|c|}
\hline GO ID & GO term & Count & $\%$ & P-value & Genes \\
\hline GO:0008009 & Chemokine activity & 11 & 11.96 & $1.69 \times 10^{-14}$ & $\begin{array}{l}\text { CXCL1, CCL12, CCL3, CCL2, CCL21C, } \\
\text { CCL9, CCL21A, PF4, CCL4, CCL7, CXCL10 }\end{array}$ \\
\hline GO:0005125 & Cytokine activity & 12 & 13.04 & $4.78 \times 10^{-9}$ & $\begin{array}{l}\text { CXCL1, CCL12, CCL3, CCL2, CCL9, PF4, } \\
\text { CCL4, CCL7, SPP1, TIMP1, CXCL10, IL11 }\end{array}$ \\
\hline GO:0048020 & CCR chemokine receptor binding & 5 & 5.44 & $8.52 \times 10^{-6}$ & CCL3, CCL21C, CCL9, CCL21A, CCL4 \\
\hline GO:0031727 & CCR2 chemokine receptor binding & 3 & 3.26 & $1.30 \times 10^{-4}$ & CCL12, CCL2, CCL7 \\
\hline GO:0008201 & Heparin binding & 4 & 4.35 & $3.43 \times 10^{-2}$ & CCL2, PF4, CCL7, CXCL10 \\
\hline GO:0020037 & Heme binding & 4 & 4.35 & $4.94 \times 10^{-2}$ & HBA-A1, CYBA, HMOX1, IDO1 \\
\hline
\end{tabular}

GO, Gene Ontology; DEGs, differentially expressed genes.

Fig. 3, which contains 67 nodes and 281 edges. Each node represents a DEG and each edge represents a PPI between two DEGs. At 24 h post-reperfusion, 23 genes served as hub genes, and of these hub genes, CCL2 exhibited the highest degree (Fig. 3).

\section{Discussion}

In the present study, 32 DEGs at $2 \mathrm{~h}, 39 \mathrm{DEG}$ at $8 \mathrm{~h}$ and $91 \mathrm{DEGs}$ at $24 \mathrm{~h}$ post-reperfusion injury were identified between cerebral I/R and sham control samples. Previous studies have performed bioinformatics analysis to identify DEGs between MCAO models and controls $(11,20-23)$. However, to the best of our knowledge, the present study is the first to perform global gene expression profiling at three time-points following reperfusion, and the findings may lead to improvements in the understanding of the pathophysiological process of cerebral I/R injury. DEGs associated with inflammation have previously been associated with cerebral I/R injury $(20,22)$, and the results of the present study were consistent with these previous reports, indicating a persistent inflammatory response in cerebral I/R injury.

In the current study, enrichment analysis revealed that 'chemotaxis', 'chemokine activity' and 'chemokine signaling pathway' terms were significantly enriched for the obtained DEGs. Furthermore, members of the chemokine family were the most abundant among the upregulated genes that were common among 2, 8 and $24 \mathrm{~h}$ post-reperfusion 
Table V. Enriched KEGG pathways for DEGs between cerebral ischemia/reperfusion and sham control samples.

A, Enriched KEGG pathways for DEGs at $2 \mathrm{~h}$ post-reperfusion

\begin{tabular}{llllll}
\hline KEGG entry & \multicolumn{1}{c}{ Pathway name } & Count & $\%$ & P-value & Genes \\
\hline mmu05132 & Salmonella infection & 6 & 18.75 & $1.27 \times 10^{-6}$ & CXCL1, FOS, CCL3, CCL4, CD14, IL1A \\
mmu04380 & Osteoclast differentiation & 5 & 15.63 & $2.68 \times 10^{-4}$ & FOS, SOCS3, FOSB, JUNB, IL1A \\
mmu04620 & Toll-like receptor signaling pathway & 4 & 12.50 & $2.11 \times 10^{-3}$ & FOS, CCL3, CCL4, CD14 \\
mmu04062 & Chemokine signaling pathway & 4 & 12.50 & $1.34 \times 10^{-2}$ & CXCL1, CCL3, CCL4, CCL7 \\
mmu04060 & Cytokine-cytokine receptor interaction & 4 & 12.50 & $2.41 \times 10^{-2}$ & CCL3, CCL4, CCL7, IL1A \\
mmu05166 & HTLV-I infection & 4 & 12.50 & $3.35 \times 10^{-2}$ & ZFP36, FOS, EGR2, ATF3 \\
\hline
\end{tabular}

B, Enriched KEGG pathways for DEGs at $8 \mathrm{~h}$ post-reperfusion

\begin{tabular}{llrlll}
\hline KEGG entry & \multicolumn{1}{c}{ Pathway name } & Count & $\%$ & P-value & Genes \\
\hline mmu04062 & Chemokine signaling pathway & 7 & 17.95 & $2.28 \times 10^{-5}$ & CXCL1, CCL12, CCL3, CCL9, CCL4, \\
& & & & & CCL7, CCL17 \\
mmu04060 & Cytokine-cytokine receptor interaction & 6 & 15.38 & $8.01 \times 10^{-4}$ & CCL12, CCL3, FLT1, OSMR, CCL4, \\
& & & & CCL7 \\
mmu05132 & Salmonella infection & 4 & 10.26 & $1.72 \times 10^{-3}$ & CXCL1, CCL3, CCL4, CD14 \\
mmu04620 & Toll-like receptor signaling pathway & 4 & 10.26 & $3.60 \times 10^{-3}$ & CCL3, TLR2, CCL4, CD14 \\
mmu04145 & Phagosome & 4 & 10.26 & $1.61 \times 10^{-2}$ & TLR2, TUBB6, CD14, FCGR3 \\
mmu05142 & Chagas disease (American trypanosomiasis) & 3 & 7.69 & $4.02 \times 10^{-2}$ & CCL12, CCL3, TLR2
\end{tabular}

C, Enriched KEGG pathways for DEGs at $24 \mathrm{~h}$ post-reperfusion

\begin{tabular}{|c|c|c|c|c|c|}
\hline KEGG entry & Pathway name & Count & $\%$ & P-value & Genes \\
\hline mmu04062 & Chemokine signaling pathway & 11 & 11.96 & $2.50 \times 10^{-7}$ & $\begin{array}{l}\text { CXCL1, CCL12, CCL3, CCL2, CCL21C, } \\
\text { CCL9, CCL21A, PF4, CCL4, CCL7, } \\
\text { CXCL10 }\end{array}$ \\
\hline mmu04060 & Cytokine-cytokine receptor interaction & 11 & 11.96 & $1.95 \times 10^{-6}$ & $\begin{array}{l}\text { CCL12, CCL3, CCL2, TNFRSF12A, } \\
\text { CCL21C, CCL21A, PF4, CCL4, CCL7, } \\
\text { CXCL10, IL11 }\end{array}$ \\
\hline mmu05323 & Rheumatoid arthritis & 6 & 6.52 & $1.43 \times 10^{-4}$ & $\begin{array}{l}\text { CCL12, CCL3, CCL2, TLR2, MMP3, } \\
\text { IL11 }\end{array}$ \\
\hline mmu04620 & Toll-like receptor signaling pathway & 6 & 6.52 & $3.80 \times 10^{-4}$ & $\begin{array}{l}\text { CCL3, TLR2, CCL4, CD14, SPP1, } \\
\text { CXCL10 }\end{array}$ \\
\hline mmu04668 & TNF signaling pathway & 6 & 6.52 & $5.41 \times 10^{-4}$ & $\begin{array}{l}\text { CXCL1, CCL12, CCL2, SOCS3, MMP3, } \\
\text { CXCL10 }\end{array}$ \\
\hline mmu04145 & Phagosome & 7 & 7.61 & $6.73 \times 10^{-4}$ & $\begin{array}{l}\text { ACTB, CYBA, FCGR2B, FCGR4, TLR2, } \\
\text { TUBB6, CD14 }\end{array}$ \\
\hline mmu05144 & Malaria & 4 & 4.35 & $3.20 \times 10^{-3}$ & HBA-A1, CCL12, CCL2, TLR2 \\
\hline mmu05164 & Influenza A & 6 & 6.52 & $3.99 \times 10^{-3}$ & $\begin{array}{l}\text { ACTB, CCL12, CCL2, SOCS3, HSPA1A, } \\
\text { CXCL10 }\end{array}$ \\
\hline mmu05142 & Chagas disease (American trypanosomiasis) & 4 & 4.35 & $2.58 \times 10^{-2}$ & CCL12, CCL3, CCL2, TLR2 \\
\hline
\end{tabular}

KEGG, Kyoto Encyclopedia of Genes and Genomes; DEGs, differentially expressed genes.

time-points, including CCL3, CCL4, CCL7 and CXCL1. Chemokines have been reported to have complex and essential roles in I/R injury, which involves extensive leukocyte and neutrophil infiltration, subsequently exaggerating the ischemic area (24). Following ischemic stroke, chemokines are primarily produced by resident microglial cells in the brain and infiltrating immune cells, which leads to further leukocyte recruitment and activation (25). In the present study, CCL2 exhibited the highest degree in the PPI network at $24 \mathrm{~h}$ post-reperfusion, which is consistent with previous 


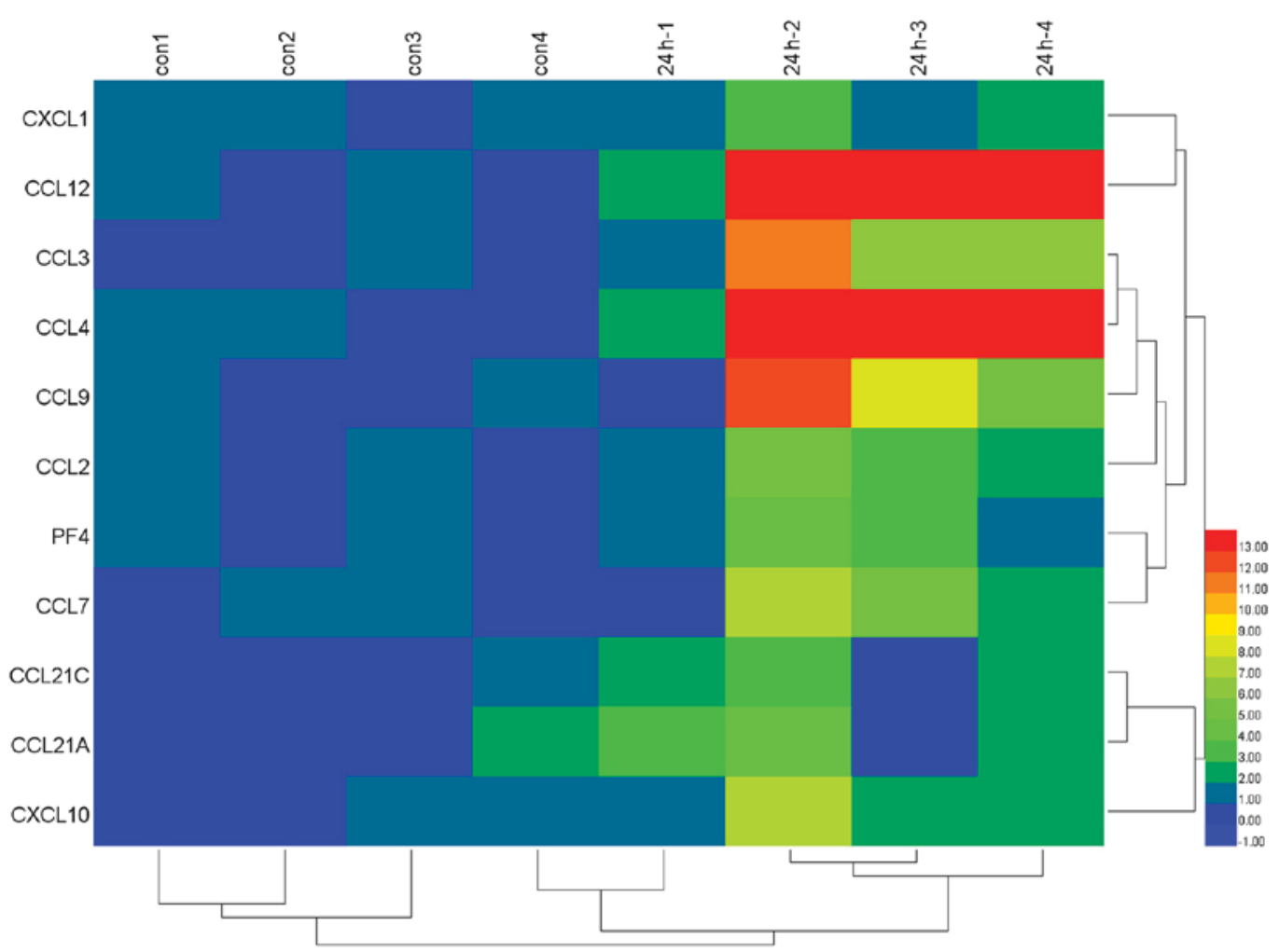

Figure 2. Heat map illustration of chemokine signaling pathway-associated genes in $24 \mathrm{~h}$ post-reperfusion and sham samples. A total of 11 chemokine signaling pathway-associated genes were included. The color code depicts the value of each gene following median normalization, with blue indicating the lowest and red as the highest value.

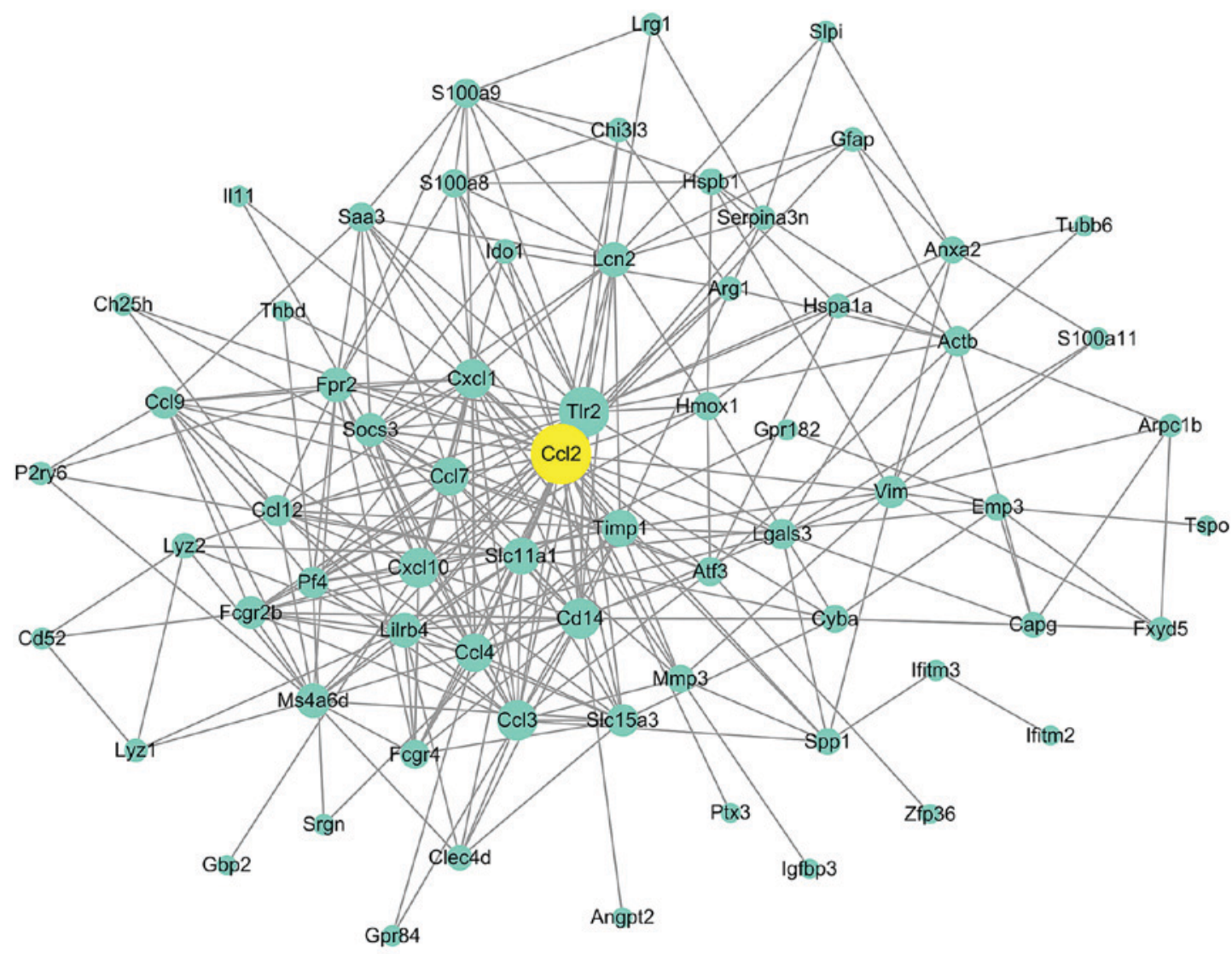

Figure 3. Protein-protein interaction network for DEGs between cerebral ischemia/reperfusion and sham control samples at $24 \mathrm{~h}$ post-reperfusion. Circles represent nodes and lines between nodes represent edges, which indicate DEGs and interactions between two DEGs, respectively. C-C motif chemokine ligand 2 was identified as the hub gene with the highest interaction degree and is indicated in yellow. DEGs, differentially expressed genes. 
Table VI. Hub genes identified in PPI networks.

$\mathrm{A}, \mathrm{Hub}$ genes in the PPI network at $2 \mathrm{~h}$ post-reperfusion

\begin{tabular}{lc}
\hline Gene & Degree \\
\hline FOS & 16 \\
CXCL1 & 11 \\
ATF3 & 11 \\
\hline
\end{tabular}

$\mathrm{B}$, Hub genes in the PPI network at $8 \mathrm{~h}$ post-reperfusion

\begin{tabular}{lc}
\hline Gene & Degree \\
\hline TLR2 & 14 \\
CXCL1 & 13 \\
CD14 & 12 \\
CCL4 & 12 \\
CCL3 & 12 \\
CCL7 & 12 \\
TIMP1 & 10 \\
\hline
\end{tabular}

$\mathrm{C}$, Hub genes in the PPI network at $24 \mathrm{~h}$ post-reperfusion

\begin{tabular}{|c|c|}
\hline Gene & Degree \\
\hline CCL2 & 37 \\
\hline TLR2 & 28 \\
\hline CCL3 & 19 \\
\hline CD14 & 19 \\
\hline CXCL10 & 19 \\
\hline CXCL1 & 18 \\
\hline CCL7 & 17 \\
\hline CCL4 & 17 \\
\hline SLC11A1 & 16 \\
\hline TIMP1 & 16 \\
\hline MS4A6D & 14 \\
\hline LCN2 & 14 \\
\hline LILRB4 & 14 \\
\hline FPR2 & 14 \\
\hline SOCS3 & 13 \\
\hline SLC15A3 & 12 \\
\hline VIM & 12 \\
\hline FCGR2B & 12 \\
\hline CCL9 & 12 \\
\hline CCL12 & 11 \\
\hline PF4 & 11 \\
\hline LGALS3 & 10 \\
\hline ACTB & 10 \\
\hline
\end{tabular}

PPI, protein-protein interaction.

studies $(26,27)$. Accordingly, CCL2 mRNA expression was initially increased at $6 \mathrm{~h}$ post-reperfusion, peaking 2 days later. Additionally, CCL3 was previously described to be upregulated post-I/R injury via the induction of monocyte accumulation in the ischemic brain $(28,29)$, and the expression of CCL3 post-reperfusion has been reported to be time-dependent (30). CCL7, as a mast cell-derived product, has been reported to be involved in the recruitment of inflammatory cells into the ischemic sites (31), subsequently contributing to stroke pathology (32). CXCL1, identified as a hub gene in the PPI networks at 2, 8 and 24 h post-reperfusion in the present study, was reported to be increased in the cerebrospinal fluid of patients that have suffered from a stroke (33). However, both neurotoxic and neuroprotective effects have been demonstrated for chemokines in post-stroke inflammation (28).

It is established that inflammation is a major contributor to stroke pathophysiology, and the immune system has been implicated in all stages of the ischemic cascade, from the acute damaging events to the progression of tissue repair (34). Microglia cells, which are closely associated with inflammation, were reported to become rapidly activated following ischemia (35). Furthermore, various pro-inflammatory factors, including interleukin (IL)-1 $\beta$, IL-6, tumor necrosis factor- $\alpha$, reactive oxygen species, nitric oxide and prostaglandin E2, were reported to be produced by activated microglia and contribute to neuronal death in cerebral ischemia (36). In the present study, specific cytokines were not measured. Further studies are required to investigate the association between the chemokine family and pro-inflammatory cytokines, which further elucidate the pathophysiological process following cerebral I/R injury.

The results of the present study revealed that the toll-like receptor signaling pathway was significantly enriched at $2 \mathrm{~h}$ post-reperfusion, suggesting early transcriptional activation. TLR2, a vital factor in the inflammatory response and tissue damage, has been reported to be implicated in cerebral ischemic damage (37). Microglia cells produce cytokines and chemokines following the stimulation of TLR2 (38). Furthermore, leukocyte and microglial infiltration, and neuronal death, were reported to be attenuated by TLR2 inhibition (39), indicating a potential novel therapeutic strategy.

In conclusion, the current study identified a set of DEGs that were altered between cerebral I/R injury samples and sham control samples. The findings may provide novel insight into the potential mechanisms underlying the development of cerebral I/R injury. Our future studies will be aimed at unveiling the potential diagnostic and prognostic value of these hub genes, which may ultimately aid the translation of these targets into clinical practice.

\section{Acknowledgements}

Not applicable.

\section{Funding}

The present study was supported by grants from the Projects of Medical and Health Technology Program in Zhejiang Province (grant no. 201482575) and the Projects of Technology Development Program in Hangzhou City (grant no. 20140633B66). 


\section{Availability of data and materials}

All data generated or analyzed during this study are included in this published article.

\section{Authors' contributions}

$\mathrm{XS}$ and $\mathrm{WB}$ were responsible for study design. $\mathrm{XH}, \mathrm{HJ}$ and $\mathrm{XS}$ were responsible for data acquisition, analysis, and interpretation. WB and ZY drafted the manuscript. ZY interpreted the results. All authors read and approved the final manuscript.

\section{Ethics approval and consent to participate}

Not applicable.

\section{Patient consent for publication}

Not applicable.

\section{Competing interests}

The authors declare that they have no competing interests.

\section{References}

1. Donnan GA, Fisher M, Macleod M and Davis SM: Stroke. Lancet 371: 1612-1623, 2008.

2. Go AS, Mozaffarian D, Roger VL, Benjamin EJ, Berry JD, Blaha MJ, Dai S, Ford ES, Fox CS, Franco S, et al: Heart disease and stroke statistics-2014 update: A report from the American Heart Association. Circulation 129: e28-e292, 2014.

3. Moskowitz MA, Lo EH and Iadecola C: The science of stroke: Mechanisms in search of treatments. Neuron 67: 181-198, 2010.

4. Hacke W, Kaste M, Bluhmki E, Brozman M, Dávalos A, Guidetti D, Larrue V, Lees KR, Medeghri Z, Machnig T, et al: Thrombolysis with alteplase 3 to $4.5 \mathrm{~h}$ after acute ischemic stroke. N Engl J Med 359: 1317-1329, 2008.

5. Lansberg MG, Bluhmki E and Thijs VN: Efficacy and safety of tissue plasminogen activator 3 to $4.5 \mathrm{~h}$ after acute ischemic stroke: A metaanalysis. Stroke 40: 2438-2441, 2009.

6. Jung JE, Kim GS, Chen H, Maier CM, Narasimhan P, Song YS, Niizuma K, Katsu M, Okami N, Yoshioka H, et al: Reperfusion and neurovascular dysfunction in stroke: From basic mechanisms to potential strategies for neuroprotection. Mol Neurobiol 41: 172-179, 2010.

7. Ding G, Jiang Q, Li L, Zhang L, Zhang ZG, Ledbetter KA Gollapalli L, Panda S, Li Q, Ewing JR and Chopp M: Angiogenesis detected after embolic stroke in rat brain using magnetic resonance T2*WI. Stroke 39: 1563-1568, 2008.

8. McColl BW, Allan SM and Rothwell NJ: Systemic infection, inflammation and acute ischemic stroke. Neuroscience 158: 1049-1061, 2009

9. Chamorro Á, Dirnagl U, Urra $X$ and Planas AM: Neuroprotection in acute stroke: Targeting excitotoxicity, oxidative and nitrosative stress, and inflammation. Lancet Neurol 15: 869-881, 2016

10. Chen C, Chu SF, Liu DD, Zhang Z, Kong LL, Zhou X and Chen NH: Chemokines play complex roles in cerebral ischemia. Neurochem Int 112: 146-158, 2018.

11. Liu C, Zhao L, Han S, Li J and Li D: Identification and functional analysis of MicroRNAs in mice following focal cerebral ischemia injury. Int J Mol Sci 16: 24302-24318, 2015.

12. Chen MJ, Wong CH, Peng ZF, Manikandan J, Melendez AJ, Tan TM, Crack PJ and Cheung NS: A global transcriptomic view of the multifaceted role of glutathione peroxidase-1 in cerebral ischemic-reperfusion injury. Free Radic Biol Med 50: 736-748, 2011.
13. Barrett T, Wilhite SE, Ledoux P, Evangelista C, Kim IF, Tomashevsky M, Marshall KA, Phillippy KH, Sherman PM, Holko M, et al: NCBI GEO: Archive for functional genomics data sets-update. Nucleic Acids Res 41 (Database Issue): D991-D995, 2013.

14. Pathan M, Keerthikumar S, Ang CS, Gangoda L, Quek CY, Williamson NA, Mouradov D, Sieber OM, Simpson RJ, Salim A, et al: FunRich: An open access standalone functional enrichment and interaction network analysis tool. Proteomics 15: 2597-2601, 2015.

15. Ashburner M, Ball CA, Blake JA, Botstein D, Butler H, Cherry JM, Davis AP, Dolinski K, Dwight SS, Eppig JT, et al: Gene ontology: Tool for the unification of biology. The Gene Ontology Consortium. Nat Genet 25: 25-29, 2000.

16. Altermann E and Klaenhammer TR: PathwayVoyager: Pathway mapping using the kyoto encyclopedia of genes and genomes (KEGG) database. BMC Genomics 6: 60, 2005.

17. Deng W, Wang Y, Liu Z, Cheng H and Xue Y: HemI: A toolkit for illustrating heatmaps. PLoS One 9: e111988, 2014.

18. Franceschini A, Szklarczyk D, Frankild S, Kuhn M, Simonovic M, Roth A, Lin J, Minguez P, Bork P, von Mering C and Jensen LJ: STRING v9.1: Protein-protein interaction networks, with increased coverage and integration. Nucleic Acids Res 41 (Database Issue): D808-D815, 2013

19. Kohl M, Wiese S and Warscheid B: Cytoscape: Software for visualization and analysis of biological networks. Methods Mol Biol 696: 291-303, 2011.

20. Wang C, Liu M, Pan Y, Bai B and Chen J: Global gene expression profile of cerebral ischemia-reperfusion injury in rat MCAO model. Oncotarget 8: 74607-74622, 2017.

21. Wang L, Yu Y, Yang J, Zhao X and Li Z: Dissecting Xuesaitong's mechanisms on preventing stroke based on the microarray and connectivity map. Mol Biosyst 11: 3033-3039, 2015.

22. White RE, Palm C, Xu L, Ling E, Ginsburg M, Daigle BJ, Han R, Patterson A, Altman RB and Giffard RG: Mice lacking the $\beta 2$ adrenergic receptor have a unique genetic profile before and after focal brain ischaemia. ASN Neuro 4: pii: e00096, 2012.

23. Wu L, Jiang Y, Zhu J, Wen Z, Xu X, Xu X, Xie Y, Yang L, $\mathrm{Xu}$ L, Lan W, et al: Orosomucoid1: Involved in vascular endothelial growth factor-induced blood-brain barrier leakage after ischemic stroke in mouse. Brain Res Bull 109: 88-98, 2014.

24. Chen C, Chu SF, Liu DD, Zhang Z, Kong LL, Zhou X and Chen NH: Chemokines play complex roles in cerebral ischemia. Neurochem Int 112: 146-158, 2018.

25. Réaux-Le Goazigo A, Van Steenwinckel J, Rostène W and Mélik Parsadaniantz S: Current status of chemokines in the adult CNS. Prog Neurobiol 104: 67-92, 2013.

26. Wang X, Yue TL, Barone FC and Feuerstein GZ: Monocyte chemoattractant protein-1 messenger RNA expression in rat ischemic cortex. Stroke 26: 661-666, 1995.

27. Yamagami S, Tamura M, Hayashi M, Endo N, Tanabe $H$, Katsuura Y and Komoriya K: Differential production of MCP-1 and cytokine-induced neutrophil chemoattractant in the ischemic brain after transient focal ischemia in rats. J Leukoc Biol 65: 744-749, 1999.

28. Mirabelli-Badenier M, Braunersreuther V, Viviani GL, Dallegri F, Quercioli A, Veneselli E, Mach F and Montecucco F: $\mathrm{CC}$ and $\mathrm{CXC}$ chemokines are pivotal mediators of cerebral injury in ischaemic stroke. Thromb Haemost 105: 409-420, 2011.

29. Cowell RM,Xu H, GalassoJM and SilversteinFS: Hypoxic-ischemic injury induces macrophage inflammatory protein-1alpha expression in immature rat brain. Stroke 33: 795-801, 2002.

30. Boddeke EW, Meigel I, Frentzel S, Gourmala NG, Harrison JK, Buttini M, Spleiss O and Gebicke-Härter P: Cultured rat microglia express functional beta-chemokine receptors. J Neuroimmunol 98: 176-184, 1999.

31. Wang X, Li X, Yaish-Ohad S, Sarau HM, Barone FC and Feuerstein GZ: Molecular cloning and expression of the rat monocyte chemotactic protein-3 gene: A possible role in stroke. Brain Res Mol Brain Res 71: 304-312, 1999.

32. Arac A, Grimbaldeston MA, Nepomuceno AR, Olayiwola O, Pereira MP, Nishiyama Y, Tsykin A, Goodall GJ, Schlecht U, Vogel $\mathrm{H}$, et al: Evidence that meningeal mast cells can worsen stroke pathology in mice. Am J Pathol 184: 2493-2504, 2014.

33. Losy J,Zaremba J and Skrobanski P: CXCL1 (GRO-alpha) chemokine in acute ischaemic stroke patients. Folia Neuropathol 43: 97-102, 2005

34. Iadecola $\mathrm{C}$ and Anrather J: The immunology of stroke: From mechanisms to translation. Nat Med 17: 796-808, 2011. 
35. Schilling M, Besselmann M, Leonhard C, Mueller M, Ringelstein EB and Kiefer R: Microglial activation precedes and predominates over macrophage infiltration in transient focal cerebral ischemia: A study in green fluorescent protein transgenic bone marrow chimeric mice. Exp Neurol 183: 25-33, 2003.

36. Jin R, Yang G and Li G: Inflammatory mechanisms in ischemic stroke: Role of inflammatory cells. J Leukoc Biol 87: 779-789, 2010.

37. Abe T, Shimamura M, Jackman K, Kurinami H, Anrather J, Zhou P and Iadecola C: Key role of CD36 in Toll-like receptor 2 signaling in cerebral ischemia. Stroke 41: 898-904, 2010.
38. Marsh BJ, Williams-Karnesky RL and Stenzel-Poore MP: Toll-like receptor signaling in endogenous neuroprotection and stroke. Neuroscience 158: 1007-1020, 2009.

39. Ziegler G, Freyer D, Harhausen D, Khojasteh U, Nietfeld W and Trendelenburg G: Blocking TLR2 in vivo protects against accumulation of inflammatory cells and neuronal injury in experimental stroke. J Cereb Blood Flow Metab 31: 757-766, 2011.

This work is licensed under a Creative Commons Attribution-NonCommercial-NoDerivatives 4.0 International (CC BY-NC-ND 4.0) License. 